\title{
Balancing Calories with Smartphone
}

\author{
Flavio Bertini ${ }^{1}$, Carlo Destro ${ }^{1}$, Danilo Montesi ${ }^{1}$ \\ ${ }^{1}$ Department of Computer Science and Engineering, University of Bologna, Italy
}

\begin{abstract}
Background: People gain weight when assume more calories than their body can consume. Instead, they lose weight when they consume more than what they eat. Comparing the body to a closed system, we can call calorie balance the difference between the input and output calories. However, keep a balanced diet regime is never a rewarding activity and often people give up.

Objectives: The goal is to help subjects with overweight problems: educate these people reduces the number of people that might migrate in the obese class. We propose an application to encourage healthier lifestyles, whose innovative feature is an automatic adaptive monitoring of the daily calorie balance. The system uses a familiar device and motivates the users to reach best result with the diet. Methods: The energy consumption is related to the oxygen consumption, obviously also if combined with physical activity.
\end{abstract}

\section{Correspondence to:}

Flavio Bertini

Department of Computer Science and Engineering,

Alma Mater Studiorum, University of Bologna

Address: Mura Anteo Zamboni, 7 - 40127 Bologna, Italy

E-mail: flavio.bertini2@unibo.it
The heart rate is directly related to the supply of oxygen. Using this simple relation, the heartbeats are bound to the calorie consumption.

Results: People achieve a greater awareness about food dosage and its calories weight. Moreover the application allows a more careful choice in food selection in order to not vanish the efforts made to change the lifestyles.

Conclusions: We have obtained good result with off-theshelf hardware and user friendly software solutions. The users consider the system like a game where they have to keep higher the burned calories level. A motivational application has been found to be a winning card to promote healthier lifestyles, without intimidating the user.

\section{Keywords}

Mobile Applications, Overweight, Energy Metabolism, Oxygen Consumption, Sedentary Lifestyle

EJBI 2015; 11(3):en13-en21

received: June 10, 2014

accepted: September 22, 2014

published: April 20, 2015

\section{Introduction}

With the increasing availability of food and with the decrease of physical activity, economically developed societies are experiencing a continuous increase in the percentage of overweight people. The obesity problem is often related to excessive calories contained in food as well as to a decrease in physical activity and a more sedentary lifestyle. Accordingly the population can be divided into three classes:

1. normal people: with adequate weight to body structure and age;

2. overweight people: with $5-25 \%$ of weight more than the optimal value for the structure and age;

3. obese people: over $25 \%$ of weight more than the optimal value for the structure and age.

This situation can be different in relation to the social contexts, but usually the cardinality of population in class (b) exceeds the population amount of the other two classes. Hence, more incisive actions must be implemented in the second group, also considering that people in class (c) come from class (b). The target people of our application is composed of overweight subjects within a range of $5-25 \%$ of the ideal weight for age and gender. For these subjects a change of diet jointly with an increased physical activity should be proposed. The goal is to balance caloric intake with energy consumption, but new problems arise when a new diet is started. Even if a great motivation pushes the subject to start the new regime and to make more physical activity, more and more people give up quickly. No appreciable weight-loss that comes in the short term and a sensation of excessive strictness cause (also) a sense of defeat that not help to reach the target results.

The contribution of this paper is to propose a complete and autonomous auto-adaptive system, able to monitor in a simple and effective way the calorie balance. The application presented does not consist in a medical device, but it is a motivational application based on a physical- 
medical theory 9]. The calories consumption is linearly related to oxygen consumption, that is directly tied to blood capacity and increases linearly with heart rate in standard workout conditions. Hence knowing the user's heart rate, it is possible to establish his/her calories consumption. On the other hand, the calories intake are calculated based on dishes that the user marks as eaten through a simple and intuitive interfaces. With these two kinds of complementary information it is possible to calculate, display and continuously update the input/output calories balance in order to give a rapid feedback whenever the user wants. Thanks to the continuous detection of the cardiac parameter of the subject, the smartphone app can calculate with a good approximation the energy expenditure of the user. Then, knowing the quantity and the type of eaten food, it is possible to inform the user about his lifestyle caloric balance. The system presents an auto-adaptive methods able to increase accuracy and keep always updated the metabolic model.

Thanks to the growth of the mobile device market, smartphone applications for health care are going to be widely used. The other systems which aim to help the user in tracking his/her calorie balance, ground their calculations only on what the user insert. Typically neither verifications nor adjustments are carried out to verify if the user inserts correct values or if the user metabolism does not correspond to the standard adopted one. These approach can show inaccurate values to the user that can lead him/her to erroneous countermeasures while trying to lose weight. Our system improves this issue using an algorithm able to compensate any change in the user lifestyle.

The outline of the paper is as follows. Section 2 specifies better the objectives of our work with some hints about the human metabolism, compared with other experience. Section 3 shows how the system works and all its features. The paper ends with some results in Session 4, a brief discussion on issues about certificates for medical devices in Session 5 and conclusions in Section 6.

\section{Objectives}

The cardinality of the overweight people group is greater than the other sets, so the main goal is to help the people of that class. It is reasonable to think that if we educate overweight people we can reduce the number of people that might migrate in the obese class. We decided to design and develop an application for the automatic auto-adaptive monitoring of the daily calorie balance. It uses a familiar device for the user and helps them to reach best result with the diet. Moreover, the system supports the user in a continued way in order to avoid an early abandonment of the diet for example because the results are slow in coming.

How to measure continuously the heartbeat and how to bind it to the burned calories in a consistent way were the first problems. The target was to avoid too complex evaluation methods that could be not accessible on a large scale, for instance as the Benedict-Roth apparatus [26]. We have developed a method able to adapt day by day the metabolic rate estimation, in order to follow the changes in the user metabolism. Further important features make the application able to provide a tracking system on patient's eating habits, in order to provide feedback on "choosing a dish" or "dietary errors".

\subsection{Metabolism and Physics Principles}

The term Basal Metabolism indicates the set of all chemical reactions that occur in the body and we can distinguish two phases:

- Catabolism the set of reactions in which complex molecules are broken down into simple ones with the release of energy;

- Anabolism the set of reactions in which complex molecules are synthesized from simpler ones with the storage of energy.

These two phases of metabolism are closely linked one with the other. Catabolism reactions provide the energy and the materials needed for anabolic reactions, which the body uses to build molecules and cellular structures necessary for its survival. Three-quarters of the energy released by the nutrients oxidation is thermical energy, while the remaining quarter is mechanical energy. Any chemical reaction and underlying metabolic processes involve energy exchanges, similarly all activities carried out by the human body require energy. The concept of energy is related to the oxygen consumption, hence all human internal chemical processes use oxygen for their reactions. All human activities contribute to the total amount of burnt oxygen that a normal non agonistic body uses in everyday life.

The synthesis of new materials, anabolic processes, in overweight people are more active than catabolic ones. In summary the increase in body weight is the result of an unbalance between the energy assumed with food and the energy cost of the organic functions (internal metabolism and physical activity). To carry out the activities, the organic chemical energy is processed and made available through the metabolic processes. That phase uses oxygen as an essential reaction element: oxygen consumption is proportional to the amount of transformed energy that is taking place within the body. If energy intake is greater than the oxidation reactions can use, chemical conversion processes are activated to fix the amount of unused energy in reserve tissues (fat tissues) that increase the body weight.

To monitor the changes in body weight, without interacting with mechanisms of assimilation and transformation, there are only two alternatives:

1. limit the energy intake through food;

2. increase the energy consumption through physical and mental activity. 
For our work it has been crucial to emphasize that the energy consumption is directly related to the oxygen consumption and it related to the heartbeats.

\subsection{Related Works}

Usually other similar projects focus only on calculating the energy consumption [1, 18] or just on the caloric intake [2. They fail to help the user to read parameters indicating whether he/she is doing a good job with the diet or the physical activity. People who are aware of the meaning of kcal will appreciate an automatic calculation of daily calorie intake [3], but for others it will be just a number without any important meaning. As a result they might not realize that a wrong eating habit may undermine the sacrifices put in place.

The heart rate monitoring mechanism is often used to derive the calories consumption of a person [19]. In particular, thanks to the performance of the new mobile devices this technique quickly became familiar in the smartphone application universe, but rarely the available solutions are supported by a base medical-physical theory. In 20] they proposed a very interesting system but no adjustments are made to compensate the variations of the user's metabolism. In [6] they understood the desirable necessity of a continuous heart rate monitoring and the target to be not bound by the proximity to the radio base station. Hence their system communicates with the smartphone of the user, allowing him/her to move outside any predefined limited coverage areas. In 21] a bulky device is proposed to monitoring the calorie balance using a PDA, the resulting system is quite invasive and users can be unmotivated to adopt it. Several other solutions have been proposed to monitor the heart rate in a non-invasive way with a wearable ECG system [4] or a ring sensor [5]. Another interesting system is to use the camera to monitoring the heartbeat 22, but in this case the user should remember to measure it regularly and the system would not have a constant data stream. Other more precise systems [23, 24 require a periodical calibration phase to measure resting metabolic rate, using specific instrumentation. Resting metabolic rate changes over time and varies the resting energy expenditure.

As stated in 7, we should not underestimate the danger of providing incorrect details to the end users, with which they can mistakenly change their eating habits or their lifestyle. From 8 we can see that the population in Western Europe is at risk for obesity but being able to gain access to advanced technologies such as smartphones and biomedical sensors, they can easily benefit from the use of techniques of automatic control of the calories consumed. Moreover, solutions to encourage and motivate healthier lifestyles 25 can be very important, especially if they are easily available as the mobile phone applications.

\section{Methods}

The proposed application allows the user to continuously measure the number of heartbeats that he/she has during the day. It uses a chest belt to calculate the energy consumption thanks to the relationship that binds oxidation reaction with oxygen consumption and heart rate. In standard conditions the oxygen consumption is proportional to the heart rate. The system starts using a first approximation: a default energy value is associated to a heartbeat. Then a better estimation can be reached considering the operating conditions detected by the instruments and applying weight-functions to the single beat.

This calibration is achievable by asking the user to measure its own weight and counting the total number of heartbeats in a given period. Knowing what he/she ate, it is possible to correct the first estimated value. The application adopts this algorithm to calibrate the weight of each heartbeat for the specific user in term of energy consumption. The user just has to write down the foods that consumes and his/her weight and he has to wear the chest belt. Thanks to the cardiac measurements made by the sensor, the application will calculate all the rest using the standard tables of food energy [15, 16. The user received the updated results on the smartphone display and is able to adjust his/her eating and physical habits. The concept behind this system is the possibility of being able to determine the energy expenditure of a subject knowing his/her heart activity [9].

It is really important to track in a continuous manner the users heart rate. We can achieve this purpose using a sensor with local memory or a sensor without local memory, that sends its readings to a device with storage and computational capabilities. Smartphones appear to be excellent candidates for this purpose: the user can always keep it close enough to receive the sensor signal. To preserve the battery power on both side a Bluetooth Low Energy protocol has been chosen to perform the communications. Currently the market offers many sensors with different shapes and features. A chest belt, with a Bluetooth 4.0 transmitter and an accuracy at least within three percent, is a kind of sensor that meets the need. The user can wear it as just wake up. It can feel the heartbeat thanks to two electrically conductive pads and with a simple formula it can update its internal heart rate measurement. Then it sends at regular intervals the read values to the application which resides on the user's smartphone. The smartphone application is able to show and manage every significant information in a very simple interface (Figure 1).

\subsection{Auto-adaptive Behavior}

How many calories does the user burn with a heartbeat? Is this value fixed or not? How does it change with respect to lifestyle change? Auto-adaptive behavior is the main innovative feature of the system. 


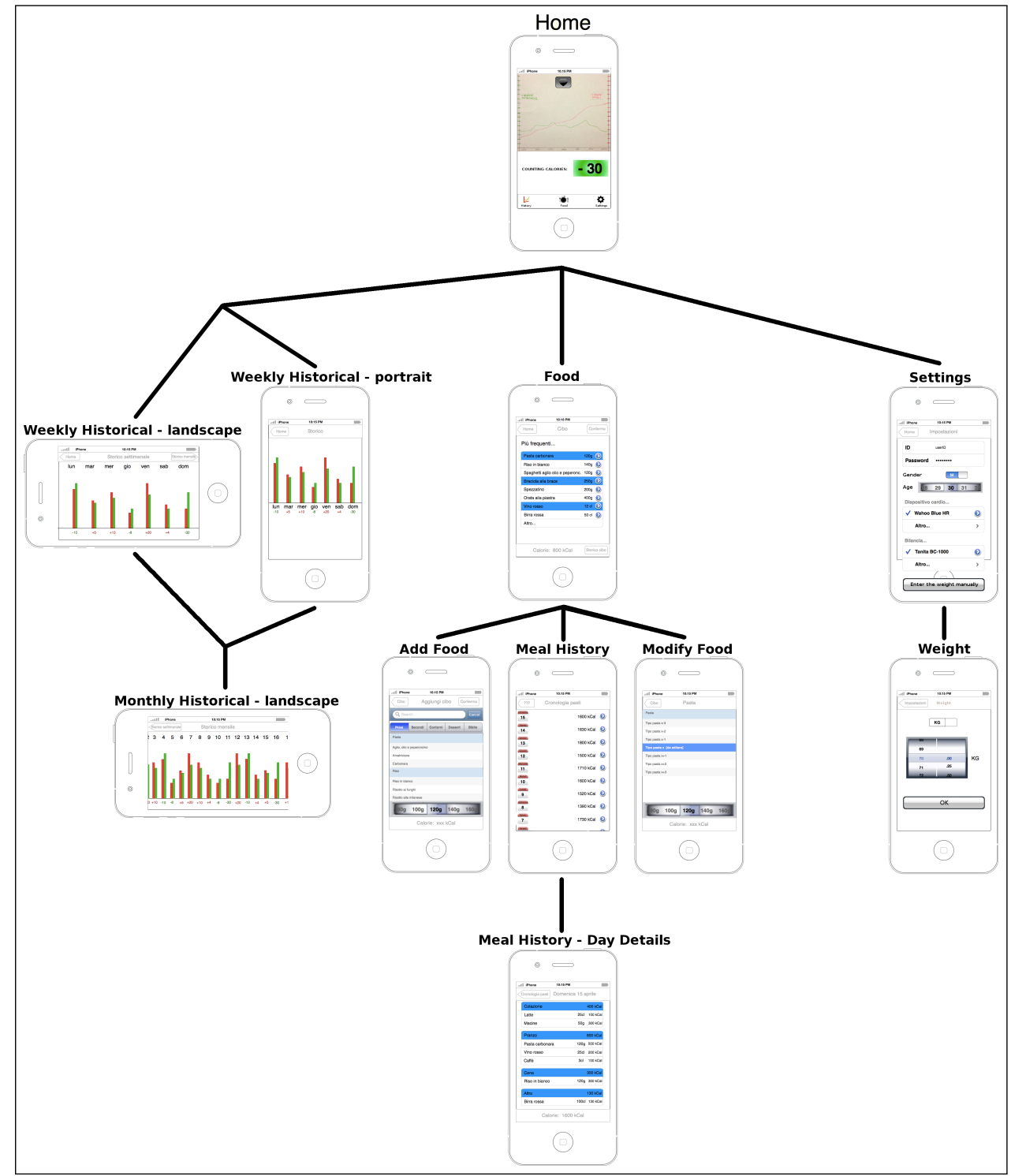

Figure 1: Application flowmap.

\subsection{A Common Approach}

The estimated "calorie weight" for each heartbeat can be calculated by a specialist, for instance with a BenedictRoth spirometer. This measure, although very faithful to the real relationship between heart rate and calories burned, refers to a particular time of the year and to a particular physical state of the subject. These conditions can change, even considerably if the subject follows a diet. Hence any fixed value can invalidate the accuracy of a system like the proposed one, especially if the user metabolism evolves day by day. Moreover only few users can access to the spirometer at the same time.

Therefore it is not possible to rely on a single precise measurement. We developed another method, less precise, integrated into the application and able to follow the subject habits.

\subsection{The Auto-adaptive Approach}

No spirometers or special tools are needed, but the user inserts some data through the application interfaces. First of all it is necessary to estimate the relationship "Beats per Calorie" (BPC) and then keeping updated this value. $\mathrm{BPC}$ is the number of heartbeats required to burn one calorie. The initial estimate can be defined dividing the daily calorie needs of the subject [17] (estimated on gender, age and weight) with the number of heartbeats occurred in the first day of use. Then the BPC correction can be done when the user makes a further weighing. It is possible to derive it from the stored data, in relation to the time elapsed from the previous weighing using this formula:

$$
\Delta K=K-H B / B P C
$$

where $\Delta K$ is the delta-calorie, $\mathrm{K}$ is the calorie intake, $\mathrm{HB}$ is the heartbeats number occurred and the $\mathrm{BPC}$ is the 
previous estimate of Beats per Calorie. Assuming that the subject weight changes due to an accumulation or a loss of body fat (except great developments of muscle mass) the energy produced can be estimated in $7.7 \mathrm{kcal} / \mathrm{gr}$. Hence if the $\Delta K$ between two weighings fits this equation:

$$
\Delta W=\Delta K / 7.7 \mathrm{kcal} / \mathrm{gr}
$$

where the $\Delta W$ is the real difference of weight, it means that the initial BPC estimate is fine. Otherwise the BPC in (1) should be adjusted in order that $\Delta W$ complies with (2).

To limit damages caused by an incorrect reading or too much readings taken at a short distance, a restriction of the incisiveness of the correction formula is performed. Also because people at home usually have not accurate balance scales, the algorithm can be led to an erroneous correction if a wrong weighting result is inserted in the formula. To limit that this possibility occur, after a weighting the algorithm doesn't correct the previous BPC with $100 \%$ strength; instead throttle the correction based on how much time has passed since the previous weighing. Therefore, weighting far back in time make a strong correction on the BPC, while close weighing affect it very little.

The application initially uses a standard estimate value to calculate the calories burned, given a certain number of heartbeats. Then it starts to auto-adapt to the metabolic characteristics of the user. Using the application, the algorithm is able to correct its parameters, providing as reliable as possible the energy consumption of the user. This behavior allows the system to provide responses always accurate, despite the user's metabolism varies as a result of the new lifestyle undertaken. In addition to the benefit of "chase" the metabolic behavior of the user, the auto-adaptation capability allows the application to tolerate the possible inaccuracy of the heart rate detection system. It is useful if the user decides to use a cheaper and less precise chest belt.

In a human-computer interaction system the component more prone to errors is often the human one, our system improves this lack. The auto-adaptation function is able to tolerate the estimation errors that the user can commit when inserts the amounts of eaten food. The auto-adaptation retains its validity only in the case where this estimation error lies inside a tolerance range, otherwise the computation is repeated. The system is able to adapt to mutable variables listed above because starting from a default BPC, a consumption of calories between two user weightings is estimated according to the number of heartbeats recorded. If the amount of intaken calories is equivalent to those consumed at the time of the second weighing, the user must weigh as much as the first time. Otherwise if he/she has increased/decreased weight and the system does not detect this variation, it means that the estimation of calories applied to each beat is too low/high. In this case the application automatically corrects the previous value to bring it closer to the real value.

\subsection{Incentive Functions}

Among the peculiarities of the system there are some incentive functions. They ensure a better user experience and they help the user to keep the right food habit. These functions are designed in order to simplify the whole system.

\subsubsection{Immediate Feedback}

There are several difficulties that characterize the diet. The two causes that mostly affect the failure rate are the non-continuity and the early abandonment of the controlled diet.

This is mainly due to the fact that the human organism is slow to react to the diet and physical activity change: the first significant results are postponed for several weeks after starting the diet. This expectation can be too long for those who were not sufficiently determined. This situation leads them to a temporary or permanent abandonment of the diet regime.

One of the main features of our application is to encourage the user to continue and possibly to correct his/her diet regime. The system provides rapid feedback, calibrated on the number of calories burned or stored day by day, transmitting a simple message like "Keep it this way! You are going right!.

\subsubsection{Choosing a Dish}

For each dish the application shows the caloric weight expressed in kcal per 100 units of measurement (kcal/100gr for solids and kcal/100ml for liquids). If the user is undecided on the menu to be prepared, he/she can use this function to choose the food that better benefits to his/her diet regime. He/she can verify what he/she has already eaten in the previous meals and the calorie assumed. The application drives towards the better choice than otherwise the user probably would not perform.

\subsubsection{Understanding of Dietary Errors}

The history functions automatically annotate the eaten and burned calories. These features are very important, especially in the "Annual History" mode. The user can see if in the past he/she has fulfilled more or less the diet regime that had been proposed and which of his/her eating habits are more counterproductive. These functions allows the user to act with more accurate cuts on the foods more frequently assumed and that contributing negatively to a healthy and balanced diet regime (Figure 2 . 


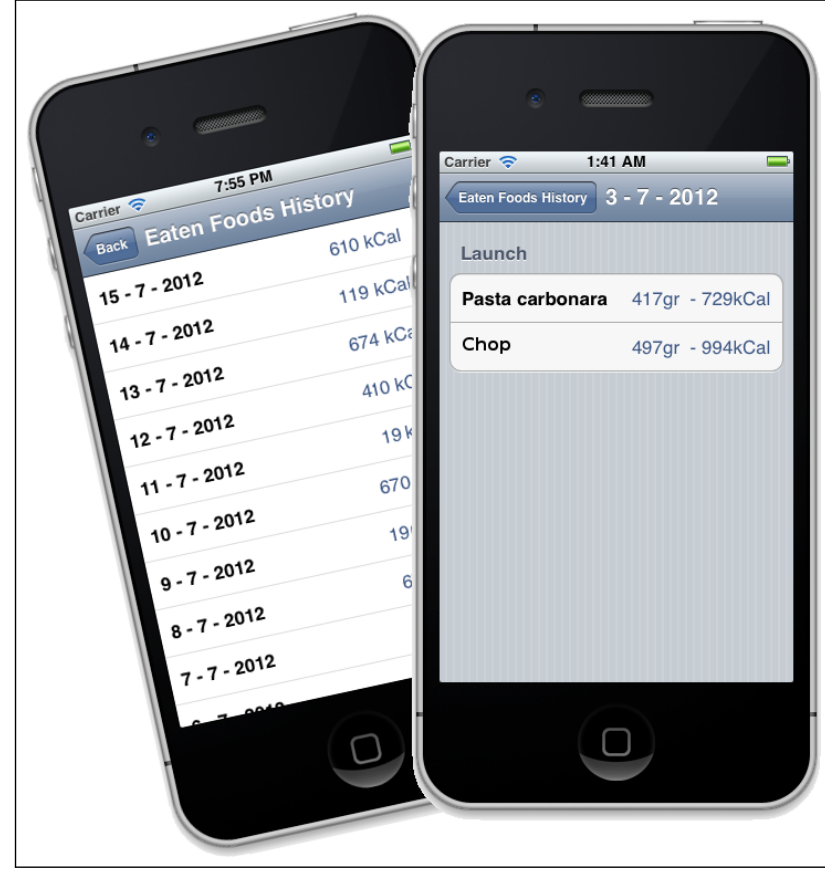

Figure 2: Details for foods eaten in the past.

\subsection{Tracking Functions}

In addition to the incentive functions a further important functionality is offered to the user. He/she can see which diet and physical workout he/she has held in the past. The Historical calories functions allows to check in the past days, weeks or months how much the user has been diligent. The Historical meals allows to understand what eating habits have impacted the most on the diet performance (Figure 3).

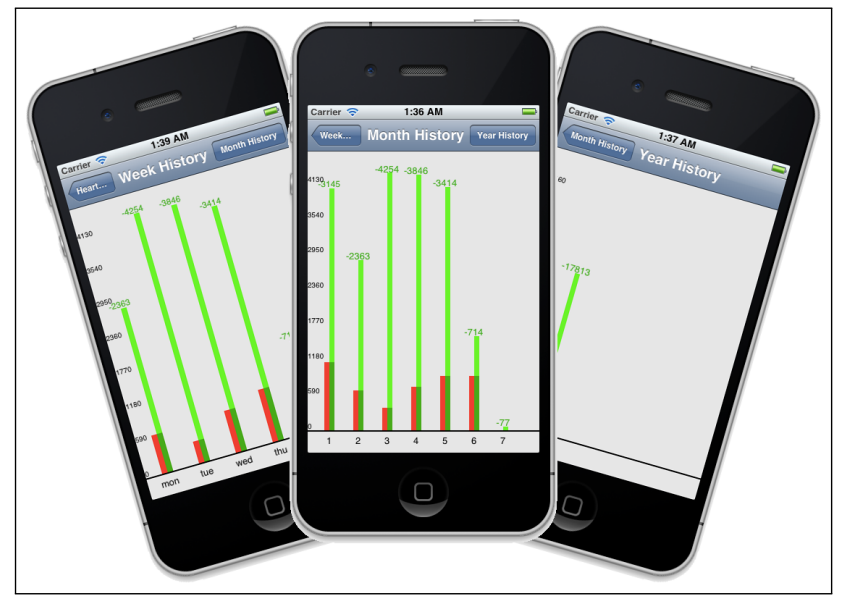

Figure 3: Details for calories balancing in past weeks, month and years.

The user is constantly informed about the danger of certain lifestyles: for instance he/she can notices an increase in weight corresponding to a time of stress or to frequent fast-food meals. If these situations will occur again in the future, the user will be able to recognize them and he will be able to react more quickly.

These storage functionality are extended also to heartbeats data. Hypothetically the doctor can be able to detect any abnormalities of the heartbeat during normal daily life, while doing sports or even during the sleeping phases.

\section{Results}

Smartphones represent the principal platforms for this kind of applications. They are the most common device currently used and they are considered by the people a familiar and multifunctional gadget. For these reasons the first experimentation of our system has been started on a iOS smartphone.

Among the results of the application it is interesting to highlight that the users have developed a greater awareness on the food's dosage and on the calorie weight of the nutrients. Moreover, the users have developed a better ability to choose dishes at time of food selection in order to not frustrate the efforts made to change their lifestyle.

Proving the system with a sample of users for a medium-long period of time, a good ease of use hes been detected by the subjects. In addition they expressed a positive opinion with respect to the type of help offered by the application with simple and intuitive graphs like the one shown in Figure 4.

We were initially doubtful about the choice of the chest belt, but the majority of the users do not feel it too intrusive. They find very useful the integration with their smartphone to which they are accustomed.

A small number of user were available to use the chest belt also during the night. Hence we could test how the system reacts if receives more information. Despite the application received the heartbeats also during the night, the error on the final computation was similar to the users that used the chest belt only during the day.

\subsection{Case Study}

In this section we propose a case study to better understand how the system works in a real situation. Let's consider a woman, as an example, Juliet: aged 30, 1.60m tall, $60 \mathrm{~kg}$ of weight, working as a teacher, so with low $/$ medium activity index.

The first day, until the first weighing, the application counts $2100 \mathrm{kcal} /$ day and assuming 100,000 heartbeats per day, the estimated BPC is 47.62. Juliet wears the chest belt daily and annotates how much she eats. After few days, the application reminds Juliet to insert a new weight. Until now the application knows neither about Juliet's metabolic consumption nor her accuracy in determine how much she eats. The new weight makes the autoadaptive algorithm able to start its work. The application knows how many heartbeats Juliet's heart did, how many calories she ate, and now also a weight difference from two 
different weighing. If the expected weight and the real weight are the same, each parameters is correctly tuned. Otherwise, if the real weight is different from the calculated one, some parameters are not correct. It can be the consequence of a poor accuracy chest belt, some mistakes made by Juliet or the BPC value used. The proposed solution implies that the auto-adaptive algorithm computes multiple parameters (also considering hour of the day, how many days passed from last weighing, connection-loss rate with the chest belt and others) and proposes a new BPC correct value. This value will be used until next weighing, when will be adjusted again. This happen for every weighing. The estimated $\mathrm{BPC}$ value becoming each time more faithful to the real one. Julia should just remember to enter the eaten foods and any mistakes that can be made in this phase will be offset by the correction of the BPC.

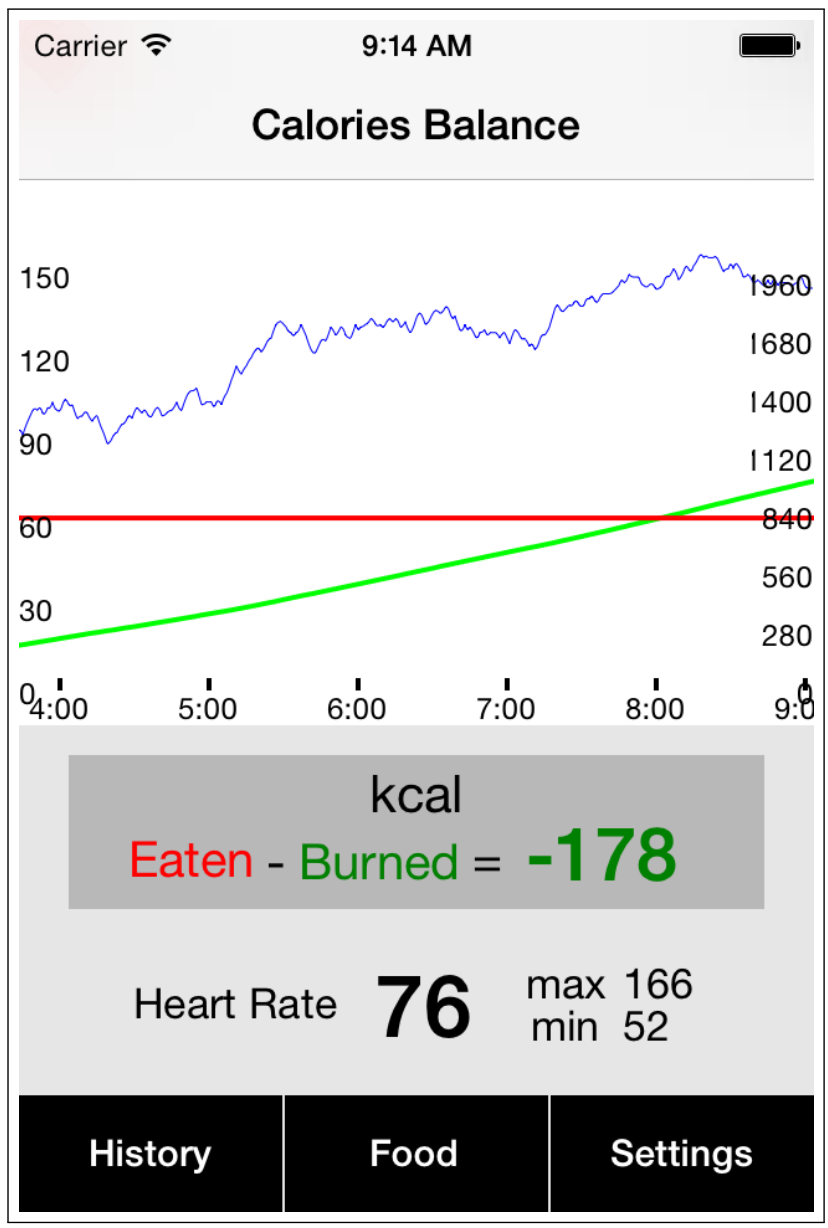

Figure 4: Details for foods eaten in the past.

Juliet can decide to wear the chest belt also during the night. However, in few days (e.g., 5 day later in this example) the application learns the Juliet lifestyle and two different situations may occur:

(a) Juliet has eaten more (or less) than she needed and has kept constant (or varied) the physical activity. As a result, she has put on weight (or lose weight).
After the weighing she annotates the new value and check $\mathrm{s}$ the situation, for instance:

(i) For 5 days she introduces $3.000 \mathrm{Kcal} /$ day, keeping 100.000 heartbeats. Her weight is grown of $585 \mathrm{gr}$.

(ii) For 5 days she introduces $1.800 \mathrm{Kcal} /$ day, but double the heartbeats with more physical activity. Her weight is decreased of $1.558 \mathrm{gr}$.

In both cases the BPC used was correct and Juliet detects the same weight variation on the balance.

(b) Juliet detects a different weight variation on the balance compared to the value indicated by the application. It means that the initial estimate BPC was not correct. In that case the application detects the error and adjusts the value for the next computation.

\section{Discussion}

Mobile applications to monitor vital signs and subject health status are definitely an interest area, so as to draw the interest of the FDA. The agency has recently published a set of guidelines [10] to improve the functionality and the interfaces of these applications. mHealth is an emerging and rapidly developing field and it is appropriate to devote a paragraph to assess the nature of the proposed system.

As defined by the European union regulations [11, mobile health covers medical and public health practice supported by mobile devices. The legislation still includes applications such as lifestyle and well being applications that may connect to medical devices or sensors. While the Italian legislation 12 transposing EU directive 13 considers a medical device an instrument used in medicine for diagnostic and/or therapeutic purposes: "any instrument, apparatus, appliance, material or other product, whether used alone or in combination, including the software necessary for its proper functioning, intended by the manufacturer to be used for human beings for the purpose of diagnosis, prevention, monitoring, treatment or alleviation of disease".

Up to now cardio chest belts are not completely considered medical devices and we do not consider the proposed application a clinical-medical device. For this reason it has not been submitted to a large-scale clinical trial, but we use a sample of users to verify the system.

The verification performed could still be useful if in a near future a certification process will be required in order to mark the system as a medical device. For us the test shown in the previous section highlights the real potential of the system. Considering the potential of these new technologies and the forecasts made by $\mathrm{PwC}$ and GSMA [14, which indicate that in 2017 mHealth could potentially save a total of $€ 99$ billion in healthcare costs in the EU, we believe that the certification path will soon be mandatory. 


\section{Conclusions}

The application presented is a motivational application that helps the user to keep a healthier lifestyle. The system has been developed using commercial software and simple hardware solutions. It makes the user able to monitor his/her cardiac performance and food habit, in order to estimate the calories balance during all day. The user inserts the amount of food that he/she ate using the various available functions and the system calculates the calories intake that these foods have led to the subject. Knowing the heart activity and the food eaten it is possible to calculate the calorie balance. In practice, it determines the difference between the calories that the person has acquired through intaken food and those who he/she consumed conducting normal activities or workout (in addition to the basal metabolism).

Then it presents the possibility for the user to access the cardiac and metabolic data and the related historical values. The user can use these information to establish the effectiveness of the diet and the lifestyle adopted. Indeed with these values the conditions with which the user can obtain most benefits are immediately identifiable and the corrections on the diet and/or on the physical activities may be taken.

The main feature of the system is the ability to autoadapt the computation, following the user lifestyle change. This characteristic protects the application from any errors that can be occur. An Incentive Function gives to the user immediate feedback on energy balance and it represents the main motivational features.

The whole system continues pushing the user to do his/her best to maintain negative the daily amount of calories stored. An exhausting commitment as a diet is tackled in a better way if it's seen as a daily game, as this application aims to do.

\section{Acknowledgements}

We would like to thank Prof. Romani Zannoli of the Department of Experimental, Diagnostic and Specialty Medicine of University of Bologna, for the idea to use the heart rate to compute the oxygen consumption and its relative burned calories.

\section{References}

[1] Pouladzadeh P, Shirmohammadi S, Arici T. Intelligent SVM based food intake measurement system. CIVEMSA 2013: Proceedings of the International Conference on Computational Intelligence and Virtual Environments for Measurement Systems and Applications; 2013 Jul 15-17; Milan, Italy. p. 87-92.

[2] Nanami R, Yoshihiro K, Tohru A. A Calorie Count Application for a Mobile Phone Based on METS Value. SECON 2008: Proceedings of the 5th Annual IEEE Communications Society Conference on Sensor, Mesh and Ad Hoc Communications and Networks; 2008 Jun 16-20; San Francisco, California, USA. p. 583-584.
[3] Pouladzadeh P, Shirmohammadi S, Al-Maghrabi R. Measuring Calorie and Nutrition From Food Image. IEEE Trans Instrum Meas [Internet]. 2014 [21 May 2014]; PP(99):1-1. Available from: http://dx.doi.org/10.1109/tim.2014.2303533

[4] Izumi S, Yamashita K, Nakano M, Konishi T, Kawaguchi H, Kimura $\mathrm{H}$ et al. A 14 ?A ECG processor with robust heart rate monitor for a wearable healthcare system. ESSCIRC 2013: Proceedings of the 39th European Solid State Circuits Conference; 2013 Sep 16-20; Bucharest, Romania. p. 145-148.

[5] Jang I, Yeom H, Sim K. Ring sensor and heart rate monitoring system for sensor network applications. Electron Lett [Internet]. 2008 [21 May 2014]; 44(24):1393 - 1394. Available from: http://dx.doi.org/10.1049/el:20082666

[6] Rotariu C, Manta V. Wireless system for remote monitoring of oxygen saturation and heart rate. FedCSIS 2012: Proceedings of the Federated Conference on Computer Science and Information Systems; 2012 Sep 9-12; Wroclaw, Poland. p. 193-196.

[7] McCartney M. How do we know whether medical apps work? BMJ [Internet]. 2013 [21 May 2014]; 346(mar20 1):f1811-f1811. Available from: http://dx.doi.org/10.1136/bmj.f1811

[8] Seidell J, Flegal K. Assessing obesity: classification and epidemiology. British medical bulletin. 1997; 53(2):238-252.

[9] Destro C. Sviluppo di applicazione per il monitoraggio della frequenza cardiaca [Undergraduate]. Department of Computer Science and Engineering Alma Mater Studiorum University of Bologna; 2012. Italian. Available from: http://amslaurea. unibo.it/3922/

[10] Department of Health and Human Services of Food and Drug Administration. Mobile medical applications: guidance for industry and Food and Drug Administration staff. 2013 Sep 25; Docket No. FDA-2011-D-0530; 2013-23293:59038-59039.

[11] European Economic Community. GREEN PAPER on mobile Health ("mHealth"). Brussels; 2014.

[12] President of the Republic. Attuazione della direttiva 93/42/CEE, concernente i dispositivi medici. Gazzetta Ufficiale n. 54 del 6 marzo 1997 - Supplemento Ordinario n. 49; 2014. Italian.

[13] European Economic Community. Council Directive 93/42/EEC of 14 June 1993 concerning medical devices. 1993.

[14] GSMA. Socio-economic impact of mHealth. PricewaterhouseCoopers; 2013.

[15] Kraisid Tontisirin., MacLean W, Warwick P. Food energy. 1st ed. Rome: Food and Agriculture Organization of the United Nations; 2003.

[16] Senser F, Souci S, Souci-Fachmann-Kraut. Der kleine SouciFachmann-Kraut. 1st ed. Stuttgart: Wiss. Verl.-Ges.; 2004.

[17] FAO, World Health Organization, United Nations University. Human energy requirements. 1st ed. Rome: FAO; 2004.

[18] Polar Electro Oy. Caloric exercise monitor. United States; US 6605044 B2, 2003.

[19] Healthetech, Inc. Monitoring caloric expenditure resulting from body activity. United States; US 6571200 B1, 2003.

[20] Lee G, Tsai C, Griswold W, Raab F, Patrick K. PmEB. CHI '06 extended abstracts on Human factors in computing systems - CHI EA '06 [Internet]. 2006 [cited 15 September 2014];. Available from: http://dx.doi.org/10.1145/1125451.1125645

[21] Healthetech, Inc., (2002). Integrated calorie management system. US6478736 B1. 
[22] Pelegris, P., Banitsas, K., Orbach, T. and Marias, K. (2010). A novel method to detect Heart Beat Rate using a mobile phone. 2010 Annual International Conference of the IEEE Engineering in Medicine and Biology. [online] Available at: http://dx.doi.org/10.1109/iembs.2010.5626580

[23] Healthetech, Inc., (2003). Diet and activity-monitoring device. US6513532 B2.

[24] Denning, T., Andrew, A., Chaudhri, R., Hartung, C., Lester, J., Borriello, G. and Duncan, G. (2009). BALANCE. Proceedings of the 10th workshop on Mobile Computing Sys- tems and Applications - HotMobile '09. [online] Available at: http://dx.doi.org/10.1145/1514411.1514416

[25] Silva, B., Lopes, I., Rodrigues, J. and Ray, P. (2011). SapoFitness: A mobile health application for dietary evaluation. 2011 IEEE 13th International Conference on e-Health Networking, Applications and Services. [online] Available at: http: //dx.doi.org/10.1109/health.2011.6026782

[26] Benedict F. Human vitality and efficiency under prolonged restricted diet. 1st ed. Washington: Carnegie institution of Washington; 1919. 